Pacific Journal of Mathematics

SIMULTANEOUS DIOPHANTINE APPROXIMATIONS AND Wuluam Wens adams 


\title{
SIMULTANEOUS DIOPHANTINE APPROXIMATIONS AND CUBIC IRRATIONALS
}

\author{
WILLIAM W. ADAMS
}

The purpose of this paper is to make some remarks concerning the best order of approximation in two-dimensional simultaneous diophantine approximations. That is, let $c_{0}$ be the infimum over all constants $c>0$ such that for every pair of real numbers $\beta_{1}, \beta_{2}$ there is an infinity of rational integers $q>0, p_{1}, p_{2}$ satisfying

(*) $\quad\left|q \beta_{1}-p_{1}\right|<(c / q)^{1 / 2},\left|q \beta_{2}-p_{2}\right|<(c / q)^{1 / 2}$.

Much research has been done on this problem, and the best values to date are

$$
\frac{2}{7} \leqq c_{0}<\frac{1}{46^{1 / 4}}
$$

due to Cassels [3] and Davenport [5] respectively. This paper will be concerned with this problem where $1, \beta_{1}, \beta_{2}$ is the basis of a real cubic number field.

Let $K$ be a totally real cubic number field. Let $\alpha_{0}, \alpha_{1}, \alpha_{2}$ be a basis of $K$ (over the rational numbers, Q). Let

$$
M=\mathbf{Z}\left\langle\alpha_{0}, \alpha_{1}, \alpha_{2}\right\rangle
$$

be the free $\mathbf{Z}$-module of rank 3 generated by $\alpha_{0}, \alpha_{1}, \alpha_{2} \quad(\mathbf{Z}=$ rational integers). Let $D_{M}>0$ be the discriminant of $M$ (see [2] for the relevant facts on number fields). Now for $\xi \in M$ we have

$$
\xi=\alpha_{0} x+\alpha_{1} y+\alpha_{2} z
$$

for integers $x, y, z$, so $\mathbf{N} \xi$ ( $\mathbf{N}$ denotes the "norm" of $K / \mathbf{Q}$ ) defines a ternary cubic form with rational coefficients. Define

$$
m_{+}(M)=\inf _{\substack{\xi \in M \\ \xi>0 \\ \mathbf{N} \leqslant>0}} \mathbf{N} \xi
$$

and

$$
m_{-}(M)=\inf _{\substack{\xi \in M \\ \xi>0 \\ \mathbb{N} \leqslant<0}}|\mathbf{N} \xi|
$$

Set

$$
C_{0}^{2}=\sup _{K, M} \frac{4 m_{+}(M) m_{-}(M)}{D_{M}}
$$


where the supremum is over all totally real cubic fields $K$ and all (full) modules $M$ contained in $K$.

THeOREM 1. Let $c>C_{0}$ be given. Then for all $\beta_{1}, \beta_{2}$ such that $1, \beta_{1}, \beta_{2}$ is a basis of a real cubic number field, * has an infinite number of solutions. Conversely, if $c<C_{0}$ then there exists a pair $\beta_{1}, \beta_{2}$ such that * has only a finite number of solutions.

We note that in the first assertion of the theorem $1, \beta_{1}, \beta_{2}$ is not restricted to being the basis of a totally real field. Of course, the $\beta_{1}, \beta_{2}$ of the second assertion will be such that $1, \beta_{1}, \beta_{2}$ is the basis of a totally real field.

Corollary (Cassels [3]). Let $c<2 / 7$. Then there exists $\beta_{1}, \beta_{2}$ such that ${ }^{*}$ has only a finite number of solutions.

Proof. By Theorem 1 it suffices to show that $C_{0} \geqq 2 / 7$. Let $K$ be the cyclic field defined by $f(X)=X^{3}+X^{2}-2 X-1=0$. There is a positive root $\theta$ and a negative root $\theta^{\prime}$ of $f$. Let $M$ equal the integers of $K$. So $D_{M}=49$. We have $\mathbf{N} \theta=1$ and $\mathbf{N}\left(-\theta^{\prime}\right)=-1$. Moreover $\theta>0$ and $-\theta^{\prime}>0$ so $m_{+}(M)=m_{-}(M)=1$. Hence $C_{0}^{2} \geqq 4 / 49$ as desired.

Now we turn to what is known about $C_{0}$. We state

Conjecture. $\quad C_{0}=2 / 7$.

That is, for all $K, M, m_{+}(M) m_{-}(M) \leqq D_{M} / 49$. Although this result is true in the cases I have been able to check I have no really good evidence for its validity. I state it as above merely to have a positive statement.

One can combine a result of Chalk [4, p. 330] and one of Davenport [4, p. 61] to give an upper bound for $C_{0}$. However, the best result appears to be the one derivable from Theorem 1 and Davenport's result (1). Namely, from the second part of Theorem 1 it is clear that $C_{0} \leqq c_{0}$. Thus we have

THEOREM 2.

$$
\frac{2}{7} \leqq C_{0}<\frac{1}{46^{1 / 4}} .
$$

Hence for all totally real cubic fields $K$ and all modules $M$ contained in $K$, 


$$
m_{+}(M) m_{-}(M) \leqq \frac{D_{M}}{4 \times 46^{1 / 2}}
$$

Theorem 2 is, of course, a result about ternary cubic forms (see $[4$, p. 61]). Let $f$ be a factorizable ternary cubic form of discriminant $D(f)>0$. Write

$$
f(x)=L_{1}(x) L_{2}(x) L_{3}(x)
$$

where $L_{1}, L_{2}, L_{3}$ are real linear forms. Define

$$
\begin{gathered}
m_{+}(f)=\inf _{\substack{x \in Z^{3} \\
L_{1}(x)>0 \\
f(x)>0}} f(x) \\
m_{-}(f)=\inf _{\substack{x \in Z^{3} \\
L_{1}(x)>0 \\
f(x)<0}}|f(x)| .
\end{gathered}
$$

Then if $f(x)$ has rational coefficients and does not represent 0 nontrivially we have

$$
m_{+}(f) m_{-}(f) \leqq \frac{D(f)}{4 \times 46^{1 / 2}}
$$

(For, by [4, p. 263] $f$ is proportional to a "norm" form for a totally real cubic field.)

Also we note that any counterexample to the conjecture given above would give an improvement of Cassels' result (1). Further, if the conjecture were true then by analogy with one-dimensional diophantine approximations one would expect that $c_{0}=2 / 7$ also.

The paper is divided up as follows. In $\S 2$ we give an auxiliary result on quadratic forms needed in the proof of Theorem 1 . In $\S 3$ we give the facts needed in order to determine whether $*$ has or has not an infinite number of solutions when $1, \beta_{1}, \beta_{2}$ is a basis of a cubic field. In $\S 4$ and $\S 5$ we prove Theorem 1 when $K$ is totally real. In $\S 6$ we give a much more precise result in the case that $K$ is not totally real.

2. A result on quadratic forms. We consider two-dimensional real space $\mathbf{R}^{2}$. Let $\mathscr{B}_{r}$ be the open square

$$
\mathscr{\mathscr { B }}_{r}=\left\{(x, y) \in \mathbf{R}^{2}: \max (|x|,|y|)<r\right\}
$$

for real numbers $r>0$. We are concerned with the problem of when the curve in $\mathbf{R}^{2}$ associated with a given binary quadratic form meets $\mathscr{B}_{r}$. We consider first the trivial case.

Proposition 1. Let $Z(x, y)=a x^{2}+2 b x y+c y^{2}$ be a positive defi- 
nite binary quadratic form of discriminant $d=a c-b^{2}>0$. Let $\rho>0$ be given. Consider the curve in $\mathbf{R}^{2}$

$$
\mathscr{H}: Z(x, y)=\rho d^{1 / 2} \text {. }
$$

Then if $r^{2}>\rho / 2$ we see $\mathscr{\mathscr { C }} \cap \mathscr{B}_{r} \neq \phi$. Moreover this result is best possible, as shown by the form $Z_{0}(x, y)=x^{2}+y^{2}\left(d_{0}=1\right)$.

Proof. With $Z_{0}$ as above, suppose $\max \left(|x|^{2},|y|^{2}\right)<\rho / 2$. Then $Z_{0}(x, y)<\rho / 2+\rho / 2=\rho$ and so $(x, y)$ cannot lie on $\mathscr{C}_{0}$.

Conversely, suppose $r^{2}>\rho / 2$. It suffices to find $(x, y) \in \mathscr{K}$ such that $\max \left(x^{2}, y^{2}\right) \leqq \rho / 2$. By homogeneity we may assume $d=1$. Now for some choice of signs of $x, y$

$$
x^{2}=y^{2}=\rho /(a+2|b|+c)
$$

lies on $\mathscr{K}$. So it suffices to show that

$$
a+2|b|+c \geqq 2
$$

when $a c-b^{2}=1$. Note that $a, c>0$, since $Z$ is positive definite. By the arithmetic-geometric mean inequality we have

$$
a+c \geqq 2(a c)^{1 / 2}=2\left(1+b^{2}\right)^{1 / 2} \geqq 2(1-|b|),
$$

which gives (2).

Unfortunately, obtaining the next result is much more tedious.

Proposition 2. Let $Z(x, y)=a x^{2}+2 b x y+c y^{2}$ be an indefinite binary quadratic form with discriminant $d=a c-b^{2}<0 . \quad$ Let $\rho, \delta>0$ be given. Consider the points (curves) in $\mathbf{R}^{2}$

$$
Z(x, y)=\rho|d|^{1 / 2}
$$

$\mathscr{H}:$

$$
Z(x, y)=-\delta \rho|d|^{1 / 2} \text {. }
$$

Then if $r^{2}>\sqrt{\delta} \rho$ we have $\mathscr{P} \cap \mathscr{B}_{r} \neq \phi$. Moreover this result is best possible, as shown by the form $Z_{0}(x, y)=x^{2}-\delta y^{2}\left(d_{0}=-\delta\right)$.

Proof. With $Z_{0}$ as above suppose that $\max \left(x^{2}, y^{2}\right)<\sqrt{\delta} \rho$. Then

$$
\begin{aligned}
& x^{2}-\delta y^{2} \leqq x^{2}<\sqrt{\delta} \rho=\rho|d|^{1 / 2} \\
& x^{2}-\delta y^{2} \geqq-\delta y^{2}>-\delta \sqrt{\delta} \rho=-\delta \rho|d|^{1 / 2}
\end{aligned}
$$

so $(x, y)$ cannot lie on $\mathscr{H}_{0}$.

To prove the main part of the proposition we see we must find $(x, y) \in \mathscr{H}$ such that 


$$
\max \left(x^{2}, y^{2}\right) \leqq \sqrt{\delta} \rho
$$

The proof involves making a number of reductions and then examining many cases.

First we note that we may assume that $\delta \geqq 1$. For if $\delta<1$ set $Z^{1}=-Z, \rho^{1}=\delta \rho$ and $\delta^{1}=\delta^{-1}>1$ and we have

$$
\mathscr{H}=\mathscr{H}^{1}, \sqrt{\delta} \rho=\sqrt{\delta^{1}} \rho^{1}
$$

Now by the symmetry of the square $x \rightarrow x, y \rightarrow-y$ we may assume that $b \geqq 0$. Further, by the symmetry of the square $x \rightarrow y, y \rightarrow x$, we may assume $a<0$ implies $c<0$. Also by homogeneity we may assume $d=-1$. And finally, setting $Z^{1}=Z, \delta^{1}=\delta, \rho^{1}=1$, we see $(x, y) \in \mathscr{H}^{1}$ if and only if $\left(\rho^{1 / 2} x, \rho^{1 / 2} y\right) \in \mathscr{H}$ and so we may assume $\rho=1$.

So, to recapitulate, we have $Z(x, y)=a x^{2}+2 b x y+c y^{2}$ where

$$
\begin{aligned}
& b^{2}-a c=1, b \geqq 0 \\
& a<0 \text { implies } c<0 .
\end{aligned}
$$

Define the curves $\mathscr{H}$ by

$$
Z(x, y)=1
$$

Le:

$$
Z(x, y)=-\delta
$$

where $\delta \geqq 1$. Then we must find $(x, y) \in \mathscr{K}$ such that

$$
\max \left(x^{2}, y^{2}\right) \leqq \sqrt{\delta}
$$

Now it is readily verified that for some choices of signs of $x, y$ the values of $x^{2}, y^{2}$ listed below (under the given condition) yield points on $\mathscr{H}$.

$$
\begin{array}{ccc}
x^{2} & y^{2} & \text { Condition } \\
b^{2} /-a & -a & a<0 \\
\delta b^{2} / a & \delta a & a>0 \\
-c & b^{2} /-c & c<0 \\
\delta c & \delta b^{2} / c & c>0 \\
1 & \frac{1}{a+2 b+c} & a+2 b+c>0 \\
\frac{a+2 b+c}{a-\delta} & \frac{-\delta}{a-2 b+c} & a-2 b+c<0 .
\end{array}
$$

We show that at least one of the above six points lies on $\mathscr{Y}$ and satisfies (5). 
Case 1. Assume $a<0$. Thus by (4) we have $c<0$. Hence $a-2 b+c<0$ and point (vi) is on $\mathscr{l}$. We would be done unless

$$
-\sqrt{\delta}<a-2 b+c<0
$$

which we now assume. Now points (i), (iii) are on $\mathscr{P}$ and so it suffices to show $\max \left(b^{2} /-a,-a\right) \leqq \sqrt{\delta}$ or $\max \left(-c, b^{2} /-c\right) \leqq \sqrt{\delta}$. We have from (6) that $-a,-c \leqq \sqrt{\delta}$. Moreover we now have symmetry between $a$ and $c$, so we assume $-a \geqq-c$. Then it suffices to show that $b^{2} /-a \leqq \sqrt{\delta}$ or by (3) that $c+1 / a \geqq-\sqrt{\delta}$. Now if $-a \geqq 1$ we have from (6)

$$
c+\frac{1}{a} \geqq c+a>-\sqrt{\delta}
$$

as desired. So assume

$$
1 \geqq-a \geqq-c
$$

Now by (3) $b \geqq 1$, so by (7) $a+2 b+c>0$, so point (v) lies on $\mathscr{l}$. Thus we would be done unless

$$
0<a+2 b+c<\frac{1}{\sqrt{\bar{\delta}}}
$$

which we also assume. Now $b \geqq 1$, so (6) implies that $\sqrt{\delta} \geqq 2$. Then from (8) we have $a+c<-3 / 2$. Putting this back in (6) gives $\sqrt{\delta}>7 / 2$. Further, $a+c<-3 / 2$ and (7) yields $-a \geqq 3 / 4$. So finally

$$
c+\frac{1}{a} \geqq a+\frac{1}{a} \geqq-1-\frac{4}{3} \geqq-\sqrt{\delta}
$$

as desired.

So from now on we may assume that $a \geqq 0$.

Case 2. Assume $a+c \geqq 0$. Now if $a+c=0$ and $b=0$ we have from (3) $-a c=1$ so $a>0, c<0$ and $a=1=-c$. Point (iii) is on $\mathscr{H}$ and clearly satisfies (5), since $\delta \geqq 1$. Otherwise we have $a+2 b+c>0$, so point (vi) is on $\mathscr{H}$ and we are done unless (8) holds, which we now assume. Now if $c \geqq 0$, then (3) implies $b \geqq 1$ and this contradicts (8), since $\delta \geqq 1$. Thus $c<0$, and so $a>0$. Now we have points (ii), (iii) on $\mathscr{C}$ so it suffices to show

$$
\max \left(\frac{\delta b^{2}}{a}, \delta a\right) \leqq \sqrt{\delta} \text { or } \max \left(-c, \frac{b^{2}}{-c}\right) \leqq \sqrt{\delta} .
$$

We note first that we have $b^{2} \leqq a / \sqrt{\delta}$. This follows, since $a+c \geqq 0$ and (8) implies $b^{2}<1 / 4 \delta$. Then from (3) $1 \leqq 1 / 4 \delta-a c$. Since $a \geqq-c$ we obtain $a^{2} \geqq 1-1 / 4 \delta$. So finally 


$$
a>\left(1-\frac{1}{4 \delta}\right)^{1 / 2} \geqq \frac{1}{4 \sqrt{\delta}} \geqq \sqrt{\delta} b^{2}
$$

(since $\delta \geqq 1$ ) as desired. Therefore to prove (9) it suffices to show that $a>1 / \sqrt{\delta}$ implies $b^{2} \leqq-\sqrt{\delta} c \leqq \delta$. Well $1=b^{2}-a c \geqq a(-c)$ implies $a(-c) \leqq 1<\sqrt{\delta} a$ implies $-c<\sqrt{\delta}$. Further, by (8) and $a>1 / \sqrt{\delta}$ we have $2 b+c<0$, so

$$
b^{2}<c^{2} / 4<-\sqrt{\delta} c
$$

since $-c<4 \sqrt{\delta}$.

Case 3. Assume $a+c<0$. Then $c<0(a \geqq 0)$. Also

$$
a-2 b+c<0,
$$

so point (vi) is on $\mathscr{X}$ and we are done unless (6) holds, which we now assume.

Subcase 1. We assume $a=0$. Thus $b=1$. Then (6) yields

$$
2-\sqrt{\delta}<c<0
$$

so $\delta>4$. Now point (iii) is on $\mathscr{C}$. Moreover $-c<\sqrt{\delta}-2<\sqrt{\delta}$. So we are done, unless

$$
-c<\frac{1}{\sqrt{\delta}}<\frac{1}{2}
$$

which we now assume. Now by (10)

$$
a+2 b+c=2+c>2-\frac{1}{2}>0,
$$

so point (v) is on $\mathscr{H}$ also. Moreover,

$$
\frac{1}{a+2 b+c}=\frac{1}{2+c}<\frac{2}{3}<\sqrt{\delta} \text {. }
$$

Subcase 2. We may now assume $a>0$. So points (ii) and (iii) are on $\mathscr{H}$ and it suffices to prove (9). We first show

$$
-c>\sqrt{\delta} \text { implies } b^{2} \leqq a / \sqrt{\delta} \leqq \frac{1}{\delta} .
$$

First by (3) $a(-c) \leqq 1$, so $-c>\sqrt{\delta}$ implies $a<1 / \sqrt{\delta}$. And by (6) and $-c>\sqrt{\delta}$ we have $2 b<a$, so $b^{2} \leqq a^{2} / 4 \leqq a / \sqrt{\delta}$, since $a<$ $4 / \sqrt{\delta}$. Therefore we may further assume $-c \leqq \sqrt{\delta}$. So to prove (9) it suffices to show $b^{2} \leqq-\sqrt{\delta} c$. Now (6) implies $b^{2}<\delta / 4$. Thus $b^{2} \leqq-c \sqrt{\delta}$ unless $-c<\sqrt{\delta} / 4$, which we assume. Then 


$$
1=b^{2}-a c<\frac{\delta}{4}+a(-c) \leqq \frac{\delta}{4}+c^{2} \leqq \frac{\delta}{4}+\frac{\delta}{16}=\frac{5 \delta}{16} ;
$$

thus $\delta \geqq 16 / 5$. Now if $a+2 b+c \leqq 0$ we have

$$
b \leqq \frac{-a-c}{2}<\frac{-c}{2},
$$

so

$$
b^{2} \leqq \frac{c^{2}}{4}<-\sqrt{\delta} c
$$

(since $-c \leqq \sqrt{\delta}$ ). So we may assume $a+2 b+c>0$, and so point (v) is on $\mathscr{H}$ and so we are done, unless (8) holds, which we now assume. If $-c \geqq 1 / \sqrt{\delta}$ we have

$$
b^{2}=1+a c<1 \leqq-\sqrt{\delta} c,
$$

as desired. Otherwise $-c<1 / \sqrt{\delta}$. Then from (8)

$$
2 b+c<a+2 b+c<1 / \sqrt{\delta},
$$

so

$$
2 b<\frac{1}{\sqrt{\delta}}-c<\frac{2}{\sqrt{\delta}}
$$

and

$$
b^{2}-a c<\frac{1}{\delta}+\left(\frac{1}{\sqrt{\delta}}\right)^{2}=\frac{2}{\delta}<1,
$$

since $-c>a$ and $\delta>2$, and this contradicts (3).

3. Some facts about cubic fields. Let $K$ be a real cubic number field. Let $1, \beta_{1}, \beta_{2}$ be a basis of $K$. In [1] we showed how to count the number of solutions to ${ }^{*}$ for sufficiently large $c$. In this section we record the results obtained in [1] which allow us to gain information concerning whether, for a given $c,^{*}$ has an infinity of solutions. All unproved statements given in this section may be found in [1].

If $\alpha \in K$, denote by $\alpha=\alpha^{(0)}, \alpha^{(1)}, \alpha^{(2)}$ the conjugates of $\alpha$.

Lemma 1. Given a basis $1, \beta_{1}, \beta_{2}$ of $K$ there is a basis $\alpha_{0}, \alpha_{1}, \alpha_{2}$ of $K$ satisfying

$$
\begin{aligned}
\alpha_{0}^{(i)}+\alpha_{1}^{(i)} \beta_{1}+\alpha_{2}^{(i)} \beta_{2}=0 & (i=1,2) \\
\kappa_{0}=\alpha_{0}+\alpha_{1} \beta_{1}+\alpha_{2} \beta_{2} & >0
\end{aligned}
$$


Conversely, given a basis $\alpha_{0}, \alpha_{1}, \alpha_{2}$ of $K$, we can find a basis $1, \beta_{1}, \beta_{2}$ of $K$ satisfying (11); (12) may be guaranteed by changing the signs of $\alpha_{0}, \alpha_{1}, \alpha_{2}$.

Now assume we have $\alpha_{0}, \alpha_{1}, \alpha_{2}, \beta_{1}, \beta_{2}$ as given in Lemma 1 with $\kappa_{0}>0$. Let

$$
M=\mathbf{Z}\left\langle\alpha_{0}, \alpha_{1}, \alpha_{2}\right\rangle \text {. }
$$

For $\xi \in M$ write $\xi=q \alpha_{0}+p_{1} \alpha_{1}+p_{2} \alpha_{2}$, and in this way we view $M$ as being in one-to-one correspondence with the possible solutions to *

Now for $i=1,2$ we have from (11)

$$
-\xi^{(i)}=\alpha_{1}^{(i)}\left(q \beta_{1}-p_{1}\right)+\alpha_{2}^{(i)}\left(q \beta_{2}-p_{2}\right) \text {. }
$$

Set $\gamma_{i}=q \beta_{i}-p_{i}(i=1,2)$ and we see $\mathbf{N} \xi=\xi Z\left(\gamma_{1}, \gamma_{2}\right)$ where $Z$ is the quadratic form

$$
Z(x, y)=\left(\alpha_{1}^{(1)} x+\alpha_{2}^{(1)} y\right)\left(\alpha_{1}^{(2)} x+\alpha_{2}^{(2)} y\right) .
$$

Let $C_{1}>0$ be large but fixed, depending only on the initial data. Consider all $\xi \in M$ (for $q>0$ ) giving rise to a solution of * with $c=C_{1}$. Then for $q>0$ large

$$
\xi=q\left(\alpha_{0}+\frac{p_{1}}{q} \alpha_{1}+\frac{p_{2}}{q} \alpha_{2}\right)=q \kappa_{0}+0\left(q^{-1 / 2}\right)
$$

(in particular $\xi>0$ ). Let $\delta_{i}=q^{1 / 2} \gamma_{i}(i=1,2)$ and thus from (14) and *

$$
Z\left(\delta_{1}, \delta_{2}\right)=q Z\left(\gamma_{1}, \gamma_{2}\right)=\kappa_{0}^{-1} \xi Z\left(\gamma_{1}, \gamma_{2}\right)+0\left(q^{-3 / 2}\right) ;
$$

that is,

$$
Z\left(\delta_{1}, \delta_{2}\right)=\kappa_{0}^{-1} \mathbf{N} \xi+0\left(q^{-3 / 2}\right) .
$$

Now the values of $\mathbf{N} \xi$ for $\xi \in M$ are a discrete set of numbers. Thus the set of curves

$$
Z(x, y)=\kappa_{0}^{-1} \mathbf{N} \xi
$$

for $\xi \in M$ form a discrete set of curves. Moreover, from (15) and * values of $\mathbf{N} \xi$ are bounded and so there are only a finite number of possible curves (16) for solutions to ${ }^{*}$. Thus what we have shown is that the solutions to

$$
\left|\delta_{1}\right|,\left|\delta_{2}\right|<C_{1}^{1 / 2}
$$

(i.e., * with $c=C_{1}$ ) lie essentially on a finite set of curves in $\mathbf{R}^{2}$ defined by (16). The key result from [1] tells us how these points are 
distributed on these curves.

Proposition 3. Let $\mathscr{F}_{r}$ be the open square of radius $r>0$ defined in $\S 2$.

(1) Suppose that for some $\xi \in M$, $\mathscr{S}_{r}$ meets (16). Then there are an infinity of solutions to $*$ with $c=r^{2}$.

(2) Conversely, suppose that for all $\xi \in M$ the closure of $\mathscr{S}_{r}$ does not meet (16). Then * has only a finite number of solutions with $c=r^{2}$.

Proof. (1) Let

$$
\eta_{i}=\left(\kappa_{0}^{-1} \xi\right)^{1 / 2} \gamma_{i} \quad(i=1,2)
$$

(for $\xi>0)$. Then

$$
Z\left(\eta_{1}, \eta_{2}\right)=\kappa_{0}^{-1} \mathbf{N} \xi
$$

that is, $\left(\eta_{1}, \eta_{2}\right)$ lies on (16). Now consider a curve (16) that meets $\mathscr{B}_{r}$ for some $\xi_{0} \in M$. Then from [1] we know that the set of all $\left(\eta_{1}, \eta_{2}\right)$ on this curve such that the corresponding $\left(\delta_{1}, \delta_{2}\right)$ satisfy $*$ for some $c=C_{1}$ sufficiently large are dense in the part of the curve lying in $\mathscr{B}_{r}$. Since for these $\xi$ the $\left(\delta_{1}, \delta_{2}\right)$ satisfy $*$ it is clear that for these $\xi, q \rightarrow \infty$. From (14) then we see that for $i=1,2,\left|\eta_{i}-\delta_{i}\right| \rightarrow 0$ as $q \rightarrow \infty$. There must then be an infinite number of pairs $\left(\delta_{1}, \delta_{2}\right)$ in $\mathscr{B}$. also, as desired.

(2) Since the set of curves (16) form a discrete family, we have by hypothesis an $r^{\prime}>r$ such that none of the curves (16) meets $\mathscr{B}_{r}$. Suppose there is an infinite number of solutions to $*$ with $c=r^{2}$. Then as above we have for the corresponding $\xi$ that $\mathbf{N} \xi$ is bounded. So an infinite number of the pairs $\left(\delta_{1}, \delta_{2}\right)$ in $\mathscr{B}_{r}$ correspond to the same curve (16). As in Case 1 we have for these $\left(\delta_{1}, \delta_{2}\right)$ that

$$
\left|\eta_{i}-\delta_{i}\right| \rightarrow 0 \quad(q \rightarrow \infty)
$$

we see that an infinite number of pairs $\left(\eta_{1}, \eta_{2}\right)$ lie in $\mathscr{B}_{r^{\prime}}$. Hence some curve (16) meets $\mathscr{\mathscr { B }}_{r^{\prime}}$. This contradiction completes the proof of Proposition 3.

We require one more formula, namely one for $\kappa_{0}$. Write

$$
Z(x, y)=a x^{2}+2 b x y+c y^{2} .
$$

Then from (13)

$$
\begin{aligned}
a & =\alpha_{1}^{(1)} \alpha_{1}^{(2)} \\
2 b & =\alpha_{1}^{(1)} \alpha_{2}^{(2)}+\alpha_{1}^{(2)} \alpha_{2}^{(1)} \\
c & =\alpha_{2}^{(1)} \alpha_{2}^{(2)} .
\end{aligned}
$$


Note that even if $K$ is not totally real the $a, b, c$ are real, since then for $\alpha \in K$ we have $\alpha^{(1)}=\overline{\alpha^{(2)}}$ (complex conjugate). Let $d=a c-b^{2}$ be the discriminant of $Z$. Clearly

$$
d=\frac{1}{4}\left(\alpha_{1}^{(1)} \alpha_{2}^{(2)}-\alpha_{1}^{(2)} \alpha_{2}^{(1)}\right)^{2} .
$$

It is readily checked that $d \neq 0$. Moreover, solving equations (11) and (12) for $\kappa_{0}$ and using the assumption $\kappa_{0}>0$, we obtain

$$
\kappa_{0}^{-1}=\frac{2|d|^{1 / 2}}{\left|D_{M}\right|^{1 / 2}}
$$

where $D_{M}=\operatorname{det}\left(\alpha_{\imath}^{(j)}\right)^{2}(i, j=0,1,2)$ is the discriminant of $M$.

We note finally that $Z$ is indefinite or positive definite depending on whether $K$ is totally real or not, respectively.

4. Proof of the first half of Theorem 1 for $K$ totally real.

We combine Propositions 2, 3 to yield the result. We in fact prove the slightly more general

Theorem 3. Let $K$ be a totally real cubic field. Let $1, \beta_{1}, \beta_{2}$ be a basis of $K / \mathbf{Q}$ and define $\alpha_{0}, \alpha_{1}, \alpha_{2}$ as in Lemma 1. Let

$$
M=\mathbf{Z}\left\langle\alpha_{0}, \alpha_{1}, \alpha_{2}\right\rangle \text {. }
$$

Then for any $c>0$ such that

$$
c^{2}>\frac{4 m_{+}(M) m_{-}(M)}{D_{M}},
$$

* has an infinity of solutions.

Proof. Let

$$
\begin{aligned}
& \rho=2 m_{+}(M) / D_{M}^{1 / 2} \\
& \delta=m_{-}(M) / m_{+}(M) .
\end{aligned}
$$

Set $r^{2}=c$. So by hypothesis

$$
r^{2}>2\left(m_{+}(M) m_{-}(M)\right)^{1 / 2} / D_{M}^{1 / 2}=\sqrt{\delta} \rho .
$$

Thus, by Proposition 2, $\mathscr{B}_{r} \cap \mathscr{H} \neq \phi$. That is, $\mathscr{P}_{r}$ intersects

$$
Z(x, y)=\frac{2|d|^{1 / 2}}{D_{M}^{1 / 2}} m_{+}(M)
$$

or

$$
Z(x, y)=-\frac{2|d|^{1 / 2}}{D_{M}^{1 / 2}} m_{-}(M)
$$


There is a $\xi \in M$ such that $\mathbf{N} \xi=m_{+}(M)$ or $\mathbf{N} \xi=-m_{-}(M)$, whichever is desired (i.e., choose $\xi$ depending on whether (23) or (24) meets $\mathscr{B}$ ). So by Case 1 of Proposition 3 we have an infinite number of solutions to $*$ with $r^{2}=c$, as desired.

5. Proof of the second half of Theorem 1 for $K$ totally real. Again we prove the slightly more general

THEOREM 4. Let $K$ be a totally real cubic field. Let $M \subseteq K$ be a full module. Let $c>0$ satisfy

$$
c^{2}<\frac{4 m_{+}(M) m_{-}(M)}{D_{M}}
$$

Then there exists a basis $\alpha_{0}, \alpha_{1}, \alpha_{2}$ of $M$ such that for the associated $\beta_{1}, \beta_{2}$ of Lemma 1 , * has only a finite number of solutions.

Proof. The proof is taken, essentially, from [6]. Define $\rho, \delta$ by (21), (22) respectively.

We first determine $\alpha_{0}, \alpha_{1}, \alpha_{2}$. Let $N>0$ be large. By a lemma of Davenport [4, p. 16] there is a basis $\alpha_{0}, \alpha_{1}, \alpha_{2}$ of $M$ satisfying

$$
\begin{aligned}
& \alpha_{1}^{(1)}=N+0\left(N^{1 / 2}\right) \\
& \alpha_{1}^{(2)}=N+0\left(N^{1 / 2}\right) \\
& \alpha_{2}^{(1)}=\sqrt{\delta} N+0\left(N^{1 / 2}\right) \\
& \alpha_{2}^{(2)}=-\sqrt{\delta} N+0\left(N^{1 / 2}\right)
\end{aligned}
$$

$(N \rightarrow \infty)$. Let $\beta_{1}, \beta_{2}$ be determined by Lemma 1 . We assume $\kappa_{0}>0$ (nothing is really altered in what follows if we replace $\alpha_{i}$ by $-\alpha_{i}$ $(i=0,1,2))$.

Define $\mathscr{X}$ as in Proposition 2, that is, by (23) and (24). Let $r^{2}=c$. Now if we show that for $N$ sufficiently large, $\overline{\mathscr{B}} r \cap \mathscr{H}=\phi$ (bar denotes closure), then $\overline{\mathscr{B}}_{r}$ does not meet (16) for any $\xi \in M$, by the definition of $m_{+}(M), m_{-}(M)$ as minimal values of $\mathbf{N} \xi$. Thus by Proposition $3, *$ has only a finite number of solutions, as desired.

It remains then only to show that $\overline{\mathscr{\mathscr { B }}}$ does not meet $\mathscr{Z}$. From the definitions (17), (18), (19) we compute

$$
\begin{aligned}
& a=N^{2}+0\left(N^{3 / 2}\right) \\
& b=0\left(N^{3 / 2}\right) \\
& c=-\delta N^{2}+0\left(N^{3 / 2}\right),
\end{aligned}
$$

and so

$$
|d|^{1 / 2}=\sqrt{\delta} N^{2}+0\left(N^{3 / 2}\right) .
$$

So suppose $(x, y) \in \overline{\mathscr{B}}$. That is, 


$$
\max \left(x^{2}, y^{2}\right) \leqq r^{2}=c<\frac{2\left(m_{+}(M) m_{-}(M)\right)^{1 / 2}}{D_{M}^{1 / 2}}=\sqrt{\delta} \rho
$$

Then

$$
\begin{aligned}
Z(x, y) & \leqq N^{2} x^{2}+0\left(\left(x^{2}+|x y|+y^{2}\right) N^{3 / 2}\right) \\
& =N^{2} x^{2}+0\left(N^{3 / 2}\right) \\
& =\delta^{-1 / 2}|d|^{1 / 2} x^{2}+0\left(N^{3 / 2}\right) \\
& <\rho|d|^{1 / 2} \quad(\text { for } N \text { large })
\end{aligned}
$$

and

$$
\begin{aligned}
Z(x, y) & \geqq-\delta N^{2} y^{2}+0\left(N^{3 / 2}\right) \\
& =-\delta^{1 / 2}|d|^{1 / 2} y^{2}+0\left(N^{3 / 2}\right) \\
& >-\delta \rho|d|^{1 / 2} \quad \text { (for } N \text { large) }
\end{aligned}
$$

Thus, $(x, y)$ cannot lie on $\mathscr{Z}$.

6. The nontotally real case.

It remains to prove Theorem 1 in the case where $K$ is not totally real. Now we showed in $\S 1$ that

$$
C_{0} \geqq \frac{2}{7} \text {. }
$$

Since $1 / 23^{1 / 2}<2 / 7$, the following theorem suffices.

THEOREM 5. Let $c>23^{-1 / 2}$. Let $1, \beta_{1}, \beta_{2}$ be a basis of a real nontotally real cubic field. Then * has an infinite number of solutions. Conversely, if $c<23^{-1 / 2}$, then there exist $\beta_{1}, \beta_{2}$ such that $1, \beta_{1}, \beta_{2}$ is a basis of a real nontotally real cubic field and * has only a finite number of solutions.

The second half of this theorem is due to Furtwängler [7]. Also Theorem 5 could be stated in the more general form of Theorems 3 and 4 (see below).

Proof. The first half of this theorem parallels the proof in $\S 4$. Let $\alpha_{0}, \alpha_{1}, \alpha_{2}$ correspond to $\beta_{1}, \beta_{2}$ as in Lemma 1 . Let $M$ be the module generated by $\alpha_{0}, \alpha_{1}, \alpha_{2}$. Set

$$
m(M)=\inf _{\substack{\xi \in M \\ \xi>0}} \mathbf{N} \xi
$$

Then it is known [4, p. 61]

$$
m(M) \leqq\left|\frac{D_{M}}{23}\right|^{1 / 2}
$$


Now in Proposition 1 set

$$
\rho=\frac{2 m(M)}{\left|D_{M}\right|^{1 / 2}}
$$

and define $\mathscr{H}$ as there. Then if

$$
r^{2}>\frac{m(M)}{\left|D_{M}\right|^{1 / 2}}
$$

we have $\mathscr{B}_{r} \cap \mathscr{H} \neq \phi$. Let $r^{2}=c$. Then by hypothesis and (25)

$$
r^{2}=c>23^{-1 / 2} \geqq \frac{m(M)}{\left|D_{M}\right|^{1 / 2}} .
$$

Now there is a $\xi \in M$ such that $\mathbf{N} \xi=m(M)$. For this $\xi$ and the formula (20) for $\kappa_{0}$ we see that $\mathscr{H}$ in Proposition 1 is the curve (16) of Proposition 3. So by Proposition 3 there is an infinite number of solutions to $*$.

The converse follows closely the proof given in $\S 5$. It uses the field generated by the real root of $X^{3}-X-1$ of discriminant $23^{1 / 2}$ in the same manner as the corollary to Theorem 1 . We use Davenport's lemma to make $Z(x, y)$ look like $N^{2}\left(x^{2}+y^{2}\right)$. We do not carry out the proof here; it is essentially carried out in [6].

\section{REFERENCES}

1. W. W. Adams, Simultaneous asymptotic diophantine approximations to a basis of a real cubic number field, J. of Number Theory, 1 (1969), 179-194.

2. Z. I. Borevich and I. R. Shafarevich, Number Theory, Academic Press, New York, 1966.

3. J. W. S. Cassels, Simultaneous diophantine approximations, J. London Math. Soc. 30 (1955), 119-122.

4. 1959.

5. H. Davenport, Simultaneous diophantine approximations, Proc. London Math. Soc. 2 (1952), 408-416.

6. H. Davenport and K. Mahler, Simultaneous diophantine approximations, Duke Math. J. 13 (1946), 105-111.

7. P. Furtwängler, Uber die simultane Approximation von Irrationalzahlen, Math. Annalen 96 (1926), 169-175.

Received October 28, 1968. This work was supported partially under NSF Grant No. GP-3990.

University of California, Berkeley

Institute for Advanced Study, Princeton 


\section{PACIFIC JOURNAL OF MATHEMATICS}

\section{EDITORS}

H. ROYDEN

Stanford University

Stanford, California

Richard Pierce

University of Washington

Seattle, Washington 98105
J. DugundJI

Department of Mathematics

University of Southern California

Los Angeles, California 90007

BASIL GORDON

University of California

Los Angeles, California 90024

\section{ASSOCIATE EDITORS}

E. F. BECKENBACH

B. H. NeUmanN

F. WOLF

K. YOSHIDA

\section{SUPPORTING INSTITUTIONS}

UNIVERSITY OF BRITISH COLUMBIA

CALIFORNIA INSTITUTE OF TECHNOLOGY

UNIVERSITY OF CALIFORNIA

MONTANA STATE UNIVERSITY

UNIVERSITY OF NEVADA

NEW MEXICO STATE UNIVERSITY

OREGON STATE UNIVERSITY

UNIVERSITY OF OREGON

OSAKA UNIVERSITY

UNIVERSITY OF SOUTHERN CALIFORNIA
STANFORD UNIVERSITY

UNIVERSITY OF TOKYO

UNIVERSITY OF UTAH

WASHINGTON STATE UNIVERSITY

UNIVERSITY OF WASHINGTON

$* * \quad * \quad *$
AMERICAN MATHEMATICAL SOCIETY
CHEVRON RESEARCH CORPORATION
TRW SYSTEMS
NAVAL WEAPONS CENTER




\section{Pacific Journal of Mathematics}

Vol. 30, No. $1 \quad$ September, 1969

William Wells Adams, Simultaneous diophantine approximations and cubic irrationals ..................................... 1

Heinz Bauer and Herbert Stanley Bear, Jr., The part metric in convex

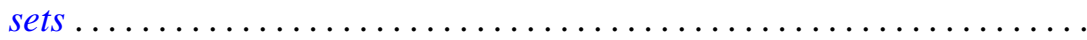

L. Carlitz, A note on exponential sums ...................... 35

Vasily Cateforis, On regular self-injective rings ................. 39

Franz Harpain and Maurice Sion, A representation theorem for measures on infinite dimensional spaces ......................... 47

Richard Earl Hodel, Sum theorems for topological spaces .............. 59

Carl Groos Jockusch, Jr. and Thomas Graham McLaughlin, Countable

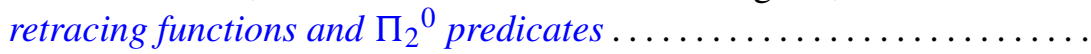

Bjarni Jónsson and George Stephen Monk, Representations of primary

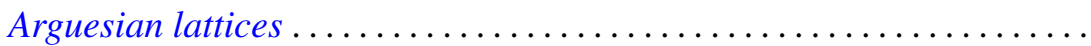

Virginia E. Walsh Knight, A continuous partial order for Peano continua...................................... 141

Kjeld Laursen, Ideal structure in generalized group algebras ........... 155

G. S. Monk, Desargues' law and the representation of primary lattices . . . 175

Hussain Sayid Nur, Singular perturbation of linear partial differential equation with constant coefficients ..........................

Richard Paul Osborne and J. L. Stern, Covering manifolds with cells ... 201

Keith Lowell Phillips and Mitchell Herbert Taibleson, Singular integrals in several variables over a local field...

James Reaves Smith, Local domains with topologically $T$-nilpotent radical....

Donald Platte Squier, Elliptic differential equations with discontinuous coefficients .................................

Tae-il Suh, Algebras formed by the Zorn vector matrix...

Earl J. Taft, Ideals in admissible algebras . .................... 259

Jun Tomiyama, On the tensor products of von Neumann algebras........ 263

David Bertram Wales, Uniqueness of the graph of a rank three group ..... 271

Charles Robert Warner and Robert James Whitley, A characterization of regular maximal ideals ......................... 